\title{
Serum Omentin Levels in Asthma Patients
}

\author{
Defne Kalaycı ${ }^{1}$, Suat Konuk ${ }^{2 *}$ and Tuncer Tuğ ${ }^{2}$ \\ ${ }^{1}$ Department of Chest Diseases, Şırnak State Hospital, Şırnak, Turkey \\ ${ }^{2}$ Department of Chest Diseases, Abant Izzel Baysal University, Bolu, Turkey
}

${ }^{*}$ Corresponding author: Dr. Suat Konuk, Department of Chest Diseases, Abant Izzel Baysal University, Bolu, Turkey, Tel: +90507 3410126 ; Email: suatkonukk@windowslive.com

Received: January 31, 2018; Accepted: February 27, 2018; Published: March 05, 2018

Citation: Kalayc D, Konuk S, Tuğ T (2018) Serum Omentin Levels in Asthma Patients. Ann Clin Lab Res Vol.6: No.1: 222.

\section{Abstract}

Background: Asthma is a heterogeneous disease, usually characterized by chronic airway inflammation. In this study, we aimed to determine the serum levels of omentin in asthma patients and its relation to atopy.

Methods: A total of 47 asthmatic patients and 39 healthy adults were involved. Patients with chronic airway disease or chronic systemic disease were not included in the study. In addition to the pateients' routine biochemical tests, the serum omentin levels were determined by the ELISA method by means of the Human Elisa Kit. The Statistical evaluation of the study was performed using the SPSS 16.0 package program and $p<0.05$ values were considered significant.

Findings: Serum omentin levels of asthmatic patients $(107.0 \pm 23.7 \mathrm{pg} / \mathrm{ml})$ were higher than those of the control group (84.0 \pm 44.2$)(p=0.028)$. A statistically significant difference was found between the serum omentin levels of the groups according to the severity of asthma $(p=0.008)$. Serum omentin levels were significantly higher only in the mild persistent asthma group compared to the moderate asthma group $(p=0.021)$. The serum omentin levels in the non-smokers were significantly higher in the control group than the smokers $(p=0.005)$. As in the asthmatic patients, also in the control group, the serum omentin levels of the women were significantly higher than the levels in men $(p<0.001)$. In asthma group, there was a negative correlation between omentin level and the body mass index in men ( $p=0.040, r=-0.599)$. In the control group, the same negative correlation was detected between omentin level and the body mass index in women $(p=0.008$, $r=-0.484)$.

Conclusion: In this study, the serum omentum levels were found to increase in asthmatic patients. In addition, it has been shown that omentum levels are higher in women than men.
Keywords: Asthma; Omentin; Adinopectin; Body mass index

\section{Introduction}

Asthma is a heterogeneous disease characterized by chronic airway inflammation.[1] Although it is known that genetic and environmental factors play a role in the formation of asthma, the etiopathogenesis has not yet been fully elucidated. Over the past 20 years there has been a marked increase in the frequency of allergic diseases, including asthma [2]. It is reported that one in four people in the American community is obese (body mass index (BMI) $\geq 30$ ) [3]. The fat tissue which was seen only as a reservoir for triglycerides and free fatty acid source is accepted today as an important part of energy metabolism by many secreted enzymes, cytokines, growth factors and hormones. Active mediators secreted from white fat tissue are called adipokines [4]. They play a role in many physiological processes of the body such as nutrition, appetite, energy balance, insulin and glucose metabolism, lipid metabolism, blood pressure regulation, vascular remodeling, coagulation and inflammation. Some of these peptides are tumor necrosis factor-alpha (TNF-alpha), interleukin-6 (IL-6), resistin, leptin, adiponectin, vaspin, visfatin and omentin. Adipokines are primarily associated with pathological processes related to obesity. In recent years, the adipokines related to various physiological mechanisms such as the immune system and inflammatory response have been described.

In this study, it was aimed to investigate omentin levels in asthmatic patients and to determine the relationship between omentin levels and inflammation severity.

\section{Materials and Methods}

This study was performed on 47 healthy asthmatic patients and 39 healthy individuals who were admitted to the Department of Chest Diseases of Izzet Baysal Training and Research Hospital between April 2015 and August 2015. The asthma patients included the patients who were both newly diagnosed and who were followed up for asthma and were in the stable or exacerbating periods of the disease. The study was started upon the approval of Instutional Ethics Board 
(Decision no. 2015/31) and Scientific Research Projects committee approval (Project no: 2015.08.28.963). The informed consent was obtained from all participants.

The participants' demographic information, smoking history, personal and relative medical history, duration and characteristics of asthma symptoms, level and severity of asthma control in the last 3 months, number of attacks in the last 1 year, symptoms of accompanying allergic diseases were recorded. A detailed questionnaire including additional disease information identified by the doctor was performed and the physical examinations were recorded. The Asthma Control Test (ACT) and the Asthma Control Questionarrie (ACQ) were performed by the physician and the scores were recorded. The patients' asthma control status was determined according to the asthma control status table in the Turkish Thoracic Society Asthma Diagnosis and Treatment Guideline 2014 Update. The height, weight measurements and physical examination findings were recorded. The respiratory function tests (MIR, Italy) were applied to asthmatic patients.

All participant's hemogram (CELL-Dyn 3700, Abbott, Illionis, USA), C-reactive protein (CRP) (latex immunoassay) and total IgE results ( ${ }^{\circledR}$ Architect c8000, Abbott, Illionis, USA) were recorded. The serum omentin levels were studied in serum samples stored at $-80^{\circ} \mathrm{C}$ in the patients and control group. The absolute eosinophil count was calculated by multiplying the percentage of eosinophils by the number of white cells. The reference upper limit values were accepted for CRP total IgE and eosinophil, respectively as $5 \mathrm{mg} / \mathrm{L}, 100 \mathrm{IU} / \mathrm{mL}$ and 400 cells $/ \mathrm{mm}^{3}$.

The serum omentin levels were studied by ELISA method (Orginal Cusabio Hubei Province, China). In addition to asthma, the patients with chronic airway disease (chronic obstructive pulmonary disease (COPD), bronchiectasis, interstitial lung disease) and/or chronic systemic disease (diabetes mellitus, liver failure, renal failure, heart failure, cerebrovascular diseases) and those younger than 18 years were excluded from the study.

SPSS 16.0 package program was used for statistical analysis. The categorical measures were given as numbers and percentages. Numeric measures were given as mean and standard deviation (where necessary, median and minimummaximum). The chi-square test was used to compare categorical measurements between groups. The normal distribution of the numerical measurements was determined by the Kolmogorov Smirnov test. The $t$ test was used in independent groups when the assumptions were met in the comparison of numerical measurements between two groups (patient-control, smoking, non-smoking, smoking, etc.), and the Mann Whitney $U$ test was used if the assumptions were not met. One-way analysis of variance was used when the assumptions were met in the comparison of numerical measurements between more than two groups, and the Kruskal Wallis test was used when the assumptions were not met. For those cases that were significant in these comparisons, the binary subgroup comparisons were made with the appropriate post-hoc tests (Scheffe, Tukey, Tamhane) or Bonferroni-corrected Mann Whitney $U$ test. The correlation between numerical measurements was examined by Pearson or Spearman correlation coefficients according to whether the assumptions were met or not. The statistical significance level was taken as $p<0.05$ in all tests.

\section{Results}

The mean age of asthmatic and control groups were $39.7 \pm$ 13.4 years and $37.2 \pm 13.8$ years, respectively. The difference was statistically insignificant $(p=0.355)$. While $35(74.5 \%)$ of the asthmatic patients were women and 12 (25.5\%) were men, in the control group, 29 patients $(74.4 \%)$ were women and 10 (25.6\%) were men $(p=0.991)$.

The serum omentin levels $(107.0 \pm 23.7 \mathrm{pg} / \mathrm{ml})$ of asthmatic patients were higher than those of the control group (84.0 \pm $44.2 \mathrm{pg} / \mathrm{ml}$ ) ( $p=0.028)$. A statistically significant difference was found when all groups were compared according to asthma severity classification $(p=0.008)$. In comparisons, the serum omentin levels were found to be higher in the mild persistent asthma group than in the moderate asthma group $(p=0.021)$. The difference between the mild persistent asthma group and the intermittent asthma group was close to the statistical significance level $(p=0.089)$.

The serum omentin levels $(111.1 \pm 21.1 \mathrm{pg} / \mathrm{ml})$ were higher in women with asthma than in men $(95.1 \pm 27.6 \mathrm{pg} / \mathrm{ml})$ $(p=0.047)$. Also, in the control group, the serum omentin levels $(98.8 \pm 38.1 \mathrm{pg} / \mathrm{ml})$ were significantly higher in women than men $(41.0 \pm 31.3 \mathrm{pg} / \mathrm{ml})$. There was no difference in serum omentin levels between smokers and non-smokers in asthma patients $(p=0.819)$. In the control group; the serum omentin level (96.6 $\pm 38.0 \mathrm{pg} / \mathrm{ml})$ was significantly higher in nonsmokers than in smokers $(51.2 \pm 44.1 \mathrm{pg} / \mathrm{ml})(\mathrm{p}=0.005)$.

The difference between the serum omentin levels in patients with and without allergy symptoms was statistically insignificant. 30 of the asthmatic patients (63.8\%) were in stable period and 17 (36.2\%) were in exacerbation period. The serum omentin levels were not significantly different between the two groups $(p=0.894)$. There was no difference in the serum omentin levels among the patients with stable asthma $(n=30)$ who were under complete control, under partial control and uncontrolled $(p=0.135)$. The serum omentin levels were found $107.3 \pm 24.5 \mathrm{pg} / \mathrm{ml}$ in patients with stable asthma whose attack number in the last one year was under 2 and $109.0 \pm 8.5 \mathrm{pg} / \mathrm{ml}$ in patients with 2 or more attacks. There was no statistical difference between the two groups $(p=0.917)$.

There was no significant correlation between the serum omentin levels and age, number of cigarettes smoked (package/year), percentage and number of neutrophils, lymphocytes, eosinophils and CRP value in both asthmatic patient and healthy control group $(p>0.05)$. In the asthmatic group, there was no significant correlation between the serum omentin level and the duration of the illness, number of attacks in the last one year, force vital capacity (FVC) and percentage, forced expiratory volume (FEV1) and its percentage, peak expiratory flow (PEF) and its percentage ( $p>0.05)$. 
There was a negative significant correlation between serum omentin level and BMI in the control group $(p=0.012$, $r=-0.399)$. There was no significant correlation between serum omentin level and BMI in asthma patients ( $p=0.125, r=-0.227)$. The negative correlations between serum omentin levels and BMI ( $p=0.040, r=-0.599)$ in men in asthma group and between serum omentin levels and BMI in women in control group $(p=0.008, r=-0.484)$ were statistically significant.

\section{Discussion}

Although the biological activity of the omentin molecule is not fully understood, recent studies have shown that many chronic inflammatory diseases are associated with increased or decreased serum omentin levels $[5,6]$. Liu et al. found in their study that in patients with carotid plaque and Type 2 DM, the serum omentin levels were lower according to the controls [7].

Zhong et al. have shown the relation of decreased omentin levels with coronary artery disease and acute coronary syndrome [8]. The patients with polycystic ovarian syndrome (PCOS) with an insulin resistance have been shown to have elevated omentin levels after treatment with metformin [9]. Shibata et al. have shown that the omentin results of 201 Japan patients showed negative correlation with metabolic risk factors such as dyslipidemia and glucose intolerance [10]. In China, as a result of a study performed on the patients with obstructive sleep apnea syndrome (OSAS), it was reported that the low serum omentin levels were associated with OSAS presence and severity [11]. In all of these studies, low omentin levels during the course of many chronic inflammatory diseases suggest that the omentin may play an antiinflammatory role in proinflammatory conditions.

To our knowledge, there is no study examining serum omentin levels in asthmatic patients. Adiponectin is an antiinflammatory adipokine such as omentin. In two studies, the low serum total adiponectin levels were found be associated with asthma in premenopausal women and peripubertal girls $[12,13]$. In other studies, conflicting results were found. Ciprandi et al. found that serum adiponectin levels were significantly higher in the pollen-induced allergic rhinitis patients than in the control group [14]. Serum adiponectin levels in symptomatic men and women patients were significantly higher in patients with seasonal allergic rhinitis than in control and asymptomatic patients [15]. Considering that there are also allergic pathways besides the inflammatory pathways in asthma pathophysiology, the high omentin levels in our study support those referenced studies.

Hsueh et al. found significantly higher serum leptin levels and lower adiponectin levels on the children with allergic rhinitis who did not have asthma [16]. There are studies that show that adipokines such as leptin show significant changes in inflammatory diseases besides the anti-inflammatory cytokines such as omentin and adiponectin [13].

In our study, there was no significant difference in mean serum omentin levels between patients with stable asthma and patients with asthma exacerbation. The serum omentin levels were not significantly different between the groups when the asthma patients were divided into groups according to the severity. CRP is a parameter that well identifies inflammation, the serum levels can be easily viewed, and systemic inflammation can also be assessed. High sensitivityCRP (Hs-CRP) values were shown to be associated with systemic inflammation in many diseases [17]. There are also studies showing the association of hs-CRP levels with asthma [18]. In a study on patients with atherosclerosis and stable angina, there was no significant relationship between serum omentin levels and hs-CRP [19]. We also found no relationship between omentin levels and hs-CRP in the asthma group. However, we found that CRP values in asthmatic patients were higher than in the healthy control group.

In this study, there was no difference in the serum omentin levels between smokers and non-smokers in the asthmatic patients. The serum omentin levels in the control group were significantly higher in smokers than in the non-smokers. Some researchers have found that the levels of brain-derived neurotrophic factor and leptin are elevated after smoking cessation $[20,21]$. Otsuka et al. have reported that the plasma adiponectin concentration increased after smoking cessation [22]. The adiponectin levels in two studies were shown to rise after smoking cessation $[23,24]$.

In this study, there was no correlation between total IgE level, eosinophil count, FEV1, FVC and serum omentin levels. Serum omentin levels were found to be significantly higher in patients with mild persistent asthma than in those with moderate and severe asthma. It is suggested that the serum omentin level may be increased by secretion as a compensatory mechanism against inflammation during the light intermittent phase.

The omentin is present in excess in human fat tissue. In thin people, the plasma omega-n levels were found to be higher than overweight and obese $[25,26]$. Thus, it was predicted that the omentin could be used as a marker for obesity-related metabolic disorders and comorbidities [26]. The plasma omentin levels were reported to be positively correlated with waist circumference, BMI, HOMA-IR (the homeostasis model assessment-estimated insulin resistance) index, plasma adiponectin and HDL-cholesterol levels. The decreased plasma omentin levels are associated with increased obesity and insulin resistance [26]. In our study, there was a negative correlation between the BMI and the omentin levels in the control group and in the asthma group in men.

There are some limitations in this study. The most important is the small number of patients. These findings need to be confirmed in larger cohorts. We think that a higher number of large prospective studies are needed to determine the position of the omentin in asthma.

\section{Conclusion}

Haematological findings have been found to be variable among different studies. Some haematological complications were observed in this study. The haemoglobin and red blood cell values were found to be reduced in HIV seropositive 
subjects both ART and non-ART compared with the controls. The explanation to this is the generalized effect of HIV/AIDS on erythropoiesis through inhibition of the precursor cells from differentiating and developing to mature red blood cells.

\section{References}

1. Busse WW, Lemanske RF (2001) Asthma. N Engl J Med 344: 350-362.

2. Fanta CH (2009) Asthma. N Engl J Med 360: 1002-1014.

3. Liu LL, Lawrence JM, Davis C, Liese AD, Pettitt DJ, et al. (2010) Prevalence of overweight and obesity in youth with diabetes in USA: the SEARCH for Diabetes in Youth study. Pediatr Diabetes 11: 4-11.

4. Kim KW, Shin YH, Lee KE, Kim ES, Sohn MH, et al. (2008) Relationship between adipokines and manifestations of childhood asthma. Pediatr Allergy Immunol 19: 535-540.

5. Schäffler A, Zeitoun $M$, Wobser $\mathrm{H}$, Buechler $\mathrm{C}$, Aslanidis $\mathrm{C}$, et al. (2007) Frequency and significance of the novel single nucleotide missense polymorphism Val109Asp in the human gene encoding omentin in Caucasian patients with type 2 diabetes mellitus or chronic inflammatory bowel diseases. Cardiovasc Diabetol 6: 3.

6. Senolt L, Polanská M, Filková M, Cerezo LA, Pavelka K, et al. (2010) Vaspin and omentin: New adipokines differentially regulated at the site of inflammation in rheumatoid arthritis. Ann Rheum Dis 69: 1410-1411.

7. Liu R, Wang X, Bu P (2011) Omentin-1 is associated with carotid atherosclerosis in patients with metabolic syndrome. Diabetes Res Clin Pract 93: 21-25.

8. Zhong X, Zhang HY, Tan H, Zhou Y, Liu FL, et al. (2011) Association of serum omentin-1 levels with coronary artery disease. Acta Pharmacol Sin 32: 873-878.

9. Tan BK, Adya R, Farhatullah S, Chen J, Lehnert H, et al. (2010) Metformin treatment may increase omentin-1 levels in women with polycystic ovary syndrome. Diabetes 59: 3023-3031.

10. Shibata R, Ouchi N, Takahashi R, Terakura Y, Ohashi K, et al. (2012) Omentin as a novel biomarker of metabolic risk factors. Diabetol Metab Syndr 4: 37-38.

11. Wang Q, Feng X, Zhou C, Li P, Kang J (2013) Decreased levels of serum omentin-1 in patients with obstructive sleep apnoea syndrome. Ann Clin Biochem 50: 230-235.

12. Sood A, Cui X, Qualls C, Beckett WS, Gross MD, et al. (2008) Association between asthma and serum adiponectin concentration in women. Thorax 63: 877-882.

13. Nagel G, Koenig W, Rapp K, Wabitsch M, Zoellner I, et al. (2009) Associations of adipokines with asthma, rhinoconjunctivitis, and eczema in German schoolchildren. Pediatr Allergy Immunol 20: 81-88.

14. Ciprandi G, Murdaca G, Marseglia G, Colombo BM, Quaglini S, et al. (2008) Serum adiponectin levels in patients with polleninduced allergic rhinitis. Int Immunopharmacol 8: 945-949.

15. Ciprandi G, De Amici M, Tosca M, Marseglia G (2010) Serum adiponectin levels in patients with seasonal allergic rhinitis. Int Immunopharmacol 10: 635-638.

16. Hsueh KC, Lin YJ, Lin HC, Lin CY (2010) Serum leptin and adiponectin levels correlate with severity of allergic rhinitis. Pediatr Allergy Immunol 21: e155-e159.

17. Tracy RP (1998) Inflammation in cardiovascular disease: Cart, horse, or both? Circulation 97: 2000-2002.

18. Takemura $M$, Matsumoto $H$, Niimi $A$, Ueda $T$, Matsuoka $H$, et al. (2006) High sensitivity C-reactive protein in asthma. The European respiratory journal 27: 908-912.

19. Yoo HJ, Hwang SY, Hong HC, Choi HY, Yang SJ, et al. (2011) Association of circulating omentin-1 level with arterial stiffness and carotid plaque in type 2 diabetes. Cardiovasc Diabetol 10: 103.

20. von der Goltz C, Koopmann A, Dinter C, Richter A, Rockenbach C, et al. (2010) Orexin and leptin are associated with nicotine craving: A link between smoking, appetite and reward. Psychoneuroendocrinology 35: 570-577.

21. Kim TS, Kim DJ, Lee H, Kim YK (2007) Increased plasma brainderived neurotrophic factor levels in chronic smokers following unaided smoking cessation. Neurosci Lett 423: 53-57.

22. Otsuka F, Kojima S, Maruyoshi H, Kojima S, Matsuzawa $Y$, et al. (2009) Smoking cessation is associated with increased plasma adiponectin levels in men. J Cardiol 53: 219-225.

23. Efstathiou SP, Skeva II, Dimas C, Panagiotou A, Parisi K, et al. (2009) Smoking cessation increases serum adiponectin levels in an apparently healthy Greek population. Atherosclerosis 205: 632-636.

24. Kotani K, Hazama A, Hagimoto A, Saika K, Shigeta M, et al. (2012) Adiponectin and smoking status: A systematic review. J Atheroscler Thromb 19: 787-794.

25. Aktaş G, Şit M, Tekçe H (2013) Yeni adipokinler: Leptin, Adiponektin ve Omentin. Abant Medical J 2:56-62.

26. Tan BK, Adya R, Farhatullah S, Lewandowski KC, O'Hare P, et al. (2008) Omentin-1, a novel adipokine, is decreased in overweight insulin-resistant women with polycystic ovary syndrome: Ex vivo and in vivo regulation of omentin-1 by insulin and glucose. Diabetes 57: 801-808. 